\title{
ÁRABE MARROQUí: DINÁMICAS DE CAMBIOS Y NUEVOS HORIZONTES
}

\author{
Adil MOUSTAOUI SRHIR* \\ Universidad Complutense de Madrid \\ Francisco MOSCOSO GARCÍA* \\ Universidad Autónoma de Madrid
}

BIBLID [1133-8571] 26 (2019) 00.1-5.

\section{Introducción.}

Durante la última década se vienen produciendo en Marruecos continuos cambios de carácter sociolingüístico. Algunos de estos cambios tienen que ver con la implantación de políticas de planificación lingüística y de estandarización del amazige, y especialmente, del árabe marroquí y que están produciendo interesantes modificaciones que afectan al estatus social, político y a la función de dichas variedades lingüísticas. Dichos cambios, que consideramos determinantes en la configuración del régimen sociolingüístico de Marruecos, generan, a su vez, reacciones y debates que van más allá de lo puramente lingüístico, siendo determinantes en la transformación de ciertas prácticas sociales, políticas, ideológicas y hasta económicas. Es decir, lejos de afectar únicamente a las «prácticas lingüísticas», las dinámicas de cambio sociolingüístico pueden y deben estudiarse también a la luz de otras transformaciones relacionadas directamente con procesos históricos de 
subordinación social y política de algunas comunidades y de las lenguas que hablan. Por esta razón, entendemos que para comprender la evolución de las relaciones de poder y desigualdad que existen en Marruecos, siendo un país multilingüe, no puede dejarse de lado el análisis de estas nuevas prácticas sociolingüísticas en árabe marroquí.

Con el deseo de responder a todas estas cuestiones, se organizó durante los días 25 y 26 de abril de 2019 el VII Congreso Internacional de árabe marroquí bajo el título Dinámicas de cambios y nuevos horizontes, en la Universidad Complutense de Madrid y en colaboración con la Universidad Autónoma de Madrid. Uno de los objetivos del Congreso fue analizar cómo se relacionan dichas dinámicas de cambio sociolingüístico, que afectan especialmente al árabe marroquí, con las nuevas prácticas lingüísticas y sociales. Del mismo modo, el objetivo del Congreso era analizar el impacto de estos cambios lingüísticos, sociales y políticos, no sólo sobre el estatus del árabe marroquí, sino también sobre los nuevos procesos de construcción identitaria que surgen a raíz de estos cambios y que están directa o indirectamente influidos por otras dinámicas de cambio.

A partir de este marco y partiendo de un enfoque sociolingüístico y de estudios culturales, todas las contribuciones al Congreso -la mayoría de ellas integran el presente monográfico- pusieron especial atención en analizar el estado actual del árabe marroquí. Algunas, se centraron en la planificación lingüística del árabe marroquí, el debate y los discursos sobre su visibilidad en la esfera pública y en el paisaje lingüístico. Igualmente, otras contribuciones abordaron aspectos relacionados con la variación lingüística en árabe marroquí, su empoderamiento social, político e identitario y las nuevas conciencias lingüísticas, no sólo en el país de origen sino también en la diáspora. Del mismo modo, interesantes contribuciones abordaron aspectos relacionados con la Literatura oral, las cuales, son un factor a tener en cuenta dentro de esta conciencia lingüística.

El resultado son veinte contribuciones que integran este monográfico y que están divididas en función de los ejes temáticos que se habían programado para el Congreso. 


\section{1. Índice y ejes temáticos}

1.1. Primer eje: El árabe marroquí en el espacio público: representaciones, discursos y paisaje

01.1-20 « De la continuité linguistique du préhilalien de type jebli au-delà du territoire des Jbala ». Fouad Brigui.

02.1-24 « Jeu de langues et paysage urbain marocain. Quelle place pour l'arabe marocain ?». Leila Messaoudi \& Mustapha Khiri.

03.1-14 « Le dialecte arabe de rhāmna (maroc) ». Issam Marjani.

04.1-13 «L'homme marocain: de la parole proverbiale à la représentation sociale ». Hamid Jaafar \& Touri Lachhab.

05.1-14 «De quelques traits préhilaliens dans le parler des ouled Boumalek / Bouhouda (Taounate) ». Rida Chalfi.

1.2. Segundo eje: Árabe marroquí y diáspora: lengua, identidad y prácticas lingüísticas

06.1-24 «Estudiantes de árabe de origen marroquí en la Universidad española: conciencia e identidad lingüística ». Francisco Moscoso García.

07.1-18 «Procesos de agentividad y nuevos repertorios translingüísticos de estudiantes de origen magrebí en Galicia». Adil Moustaoui Srhir, Gabriela Prego Vázquez \& Luz Zas Varela.

08.1-14 «El estatus nepantla del dariya ceutí: entre la bastardía y la inferiorización». Hamed Abdel-Lah

09.1-24 «El proyecto hshouma, el arte como motor de cambio social». Carmen Garratón Mateu.

1.3. Tercer eje: Estandarización y escritura del árabe marroquí

10-1-30 «Tentativas de escritura del árabe marroquí. La poesía del zéjel». Mercedes Aragón. 
11.1-14 «Moroccan Darija in Online Creation Communities: Example of Wikipedia ». Anass Sedrati \& Abderahman Ait Ali.

12.1-27 « Le théâtre à l'épreuve de l'arabe marocain ».Omar Fertat.

13.1-11 «Aproximación al estudio de las colocaciones en árabe marroquí». Houda Jaafar.

\subsection{Cuarto eje: Desafíos en la enseñanza del árabe marroquí}

14.1-16 «Árabe para todos. El desafío de elaborar materiales para la enseñanza del árabe marroquí».Victoria Aguilar Sebastián.

15.1-22 «Evolución metodológica de la enseñanza del árabe marroquí en España: del modelo tradicional a los enfoques comunicativos». Rosa Salgado Suárez.

16.1-24 «El árabe marroquí de Ceuta y el fracaso escolar: una revisión a través de la prensa local y de la investigación educativa (2005-2018)». Rafael Jiménez Gámez.

\subsection{Quinto eje: La tradición oral: entre la investigación y su enseñanza en}

\section{el aula}

17.1-23 «A cognitive approach to traditional oral poetry: frames and scripts in the jebli ayyu». Sarali Gintsburg.

18.1-19 «The characteristics of the Moroccan dialect used in traditional oral tales». Driss Cherkaoui.

19.1-20 «Cuervos y búhos: la metáfora como elemento pragmático en refranes de la dariya y del español peninsular». Carmen Vicente Molero.

20.1-19 «Les valeurs dépréciatives d'un parler de l'Oriental marocain ou le lexique du mécontentement (cas de Jerada et Ain Béni Mathar)». Mohammed Malki. 
Para completar este número monográfico hemos incluido, tras los veinte artículos de los cinco ejes, la reseña que ha realizado Adil Moustaoui Srhir del libro de Ibtissem Chachou, Sociolinguistique du Maghreb, Alger 2018, a la que hemos dado el número de orden y la paginación de 21.1-7.

2. El Congreso se celebró en el marco de los siguientes proyectos y grupos de investigación

a) Proyecto de investigación «Crisis y dinámicas locales y transnacionales en el Mediterráneo Occidental. Cambios sociopolíticos, movilizaciones y diáspora». Ref.: CSO2017-84949-C3-1-P (https://cutt.ly/Rtmbcwp)

b) Proyecto de investigación «Representaciones del islam en el Mediterráneo glocal: Cartografia e Historia conceptuales». Ref.: RTI2018-098892-BI00 (http://www.iexcul.es/proyectos.html)

c) Grupo de investigación «Ideologías y Expresiones Culturales Árabes Contemporáneas». Universidad Autónoma de Madrid, HUM-082 (www.iexcul.es).

d) Grupo de investigación «Grupo de análisis y estudio sobre Islam en Europa». Universidad Complutense de Madrid (www.ucm.es/grais).

3. La organización del Congreso contó con la financiación siguiente

a) «Ayudas para la financiación de Acciones Especiales de Investigación». Vicerrectorado de Investigación y Transferencia de la Universidad Complutense de Madrid.

b) «Ayudas para actividades culturales de la Facultad de Filosofía y Letras y del Vicerrectorado de Cooperación y Extensión Universitaria de la Universidad Autónoma de Madrid. 